\title{
Partner Notification: are Australian approaches feasible for the Chilean context?
}

\section{Iturrieta-Guaita $\mathbf{N}$, Temple-Smith $\mathbf{M} \mathbf{1}$, Tomnay $\mathbf{J}^{\mathbf{2}}$}

1 Department of General Practice, University of Melbourne

2 Department of Rural Health, Centre for Excellence in Rural Sexual Health (CERSH), University of Melbourne.

\section{Introduction:}

Partner Notification (PN) in Australia has been studied and improved in recent decades. International researchers have highlighted the use of new technology as an alternative approach for PN.(1, 2) However, more research is needed to overcome concerns about these strategies and how they can be optimised. $(3,4)$ Using the Australian experience as an example, we aim to explore clinicians' perspectives about the use of specialised websites, such as "Let them know" $\AA$ and professional counselling support, to facilitate PN in the Chilean context.

\section{Methods:}

58 semi-structured interviews were conducted with health care providers (HCP) and key informants as part of a larger qualitative study undertaken at local health services in Chile to explore PN for syphilis cases. A third of the interviews were transcribed verbatim and translated from Spanish to English for thematic analysis, which followed an inductive approach. Following the identification of themes, remaining interviews were coded utilising a method of constant comparison to highlight concordance and dissonance of participant views.

\section{Results:}
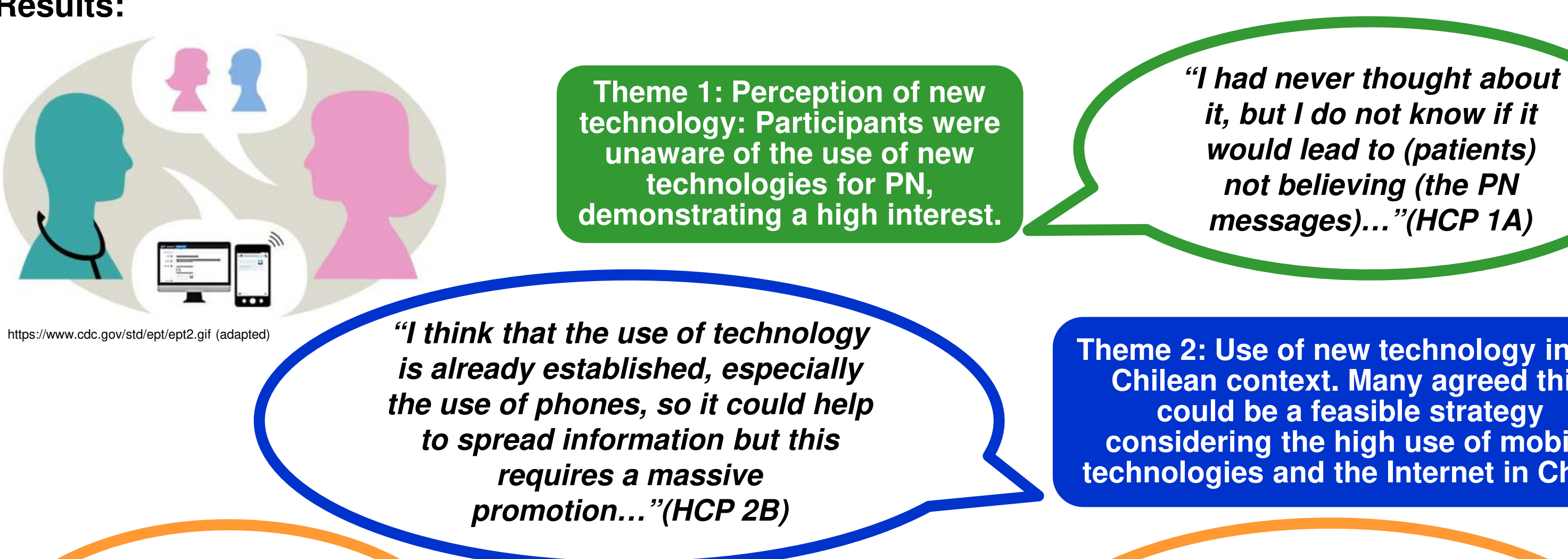

Personally, I think it is possible to misuse it or people may try to use it in a bad way ... and that may be a little complex (to deal with) (HCP 42)

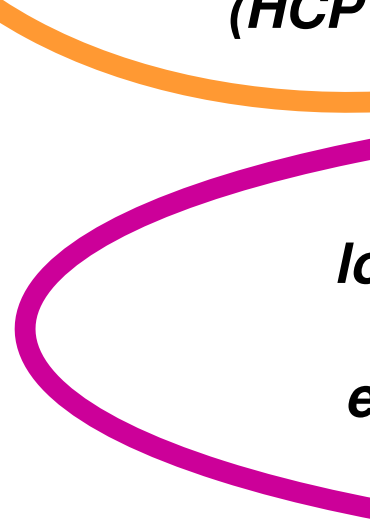

“... Having perhaps a specialised person ocally or at the health service level, who is in regular contact with clinical teams to effectively discuss how to address difficult cases..."(HCP 1)
Theme 2: Use of new technology in the Chilean context. Many agreed this could be a feasible strategy considering the high use of mobile technologies and the Internet in Chile.

"For contact tracing, I do not know whether they (new technologies) will have a good impact or not because of the impression that the population has about STIs, they remain as taboo..." (HCP 3C)

\section{Discussion:}

The use of new technologies for contacting sexual partners with professional counselling support could be an alternative PN strategy for Chile. However, the involvement of local staff will be essential in tailoring interventions.

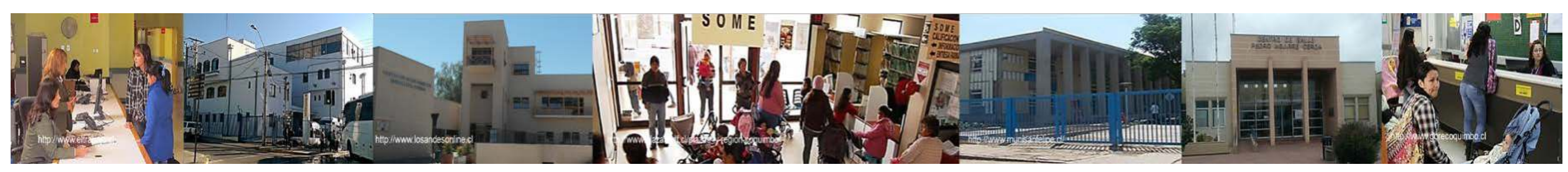

Theunissen K, et al. Barriers to and facilitators of PN for chlamydia trachomatis among health care professionals. BMC Health Services Research. 2014:14(1):492-509.

2. Pennise M, et al. Using Smartphone Apps in STD Interviews to Find Sexual Partners. Public health reports (Washington, DC: 1974). 2014:130(3):245-52.

3. Hopkins CA, et al. Telling partners about chlamydia: how acceptable are the new technologies? BMC Infectious Diseases. 2010;10(1):58-63.

4. Muessig KE, et al. Mobile Phone Applications for the Care and Prevention of HIV and Other Sexually Acknowledgements

Lilian Ferrer (PUC - Chile) and Anamaria Silva (UV - Chile), and all participants. Mrs Iturrieta-Guaita is supported by

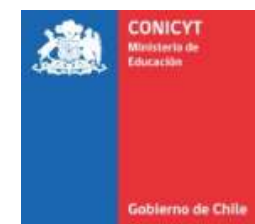

